\title{
Evaluation écologique et ethnobotanique de Jatropha curcas L. au Bénin
}

\author{
Achille Ephrem ASSOGBADJO ${ }^{1 *}$, Guillaume AMADJI ${ }^{1}$, Romain GLÈLÈ KAKAÏ ${ }^{1}$, \\ Adi MAMA ${ }^{1}$, Brice SINSIN $^{1}$, Patrick VAN DAMME ${ }^{2}$ \\ ${ }^{\text {I}}$ Faculté des Sciences Agronomiques, Université d'Abomey-Calavi, 05 BP 1752, Cotonou, Bénin. \\ ${ }^{2}$ Laboratoire d'Agriculture Tropicale et Subtropicale et d'Ethnobotanique, Université de Gand, Belgique. \\ *Auteur correspondant, E-mail : assogbadjo@yahoo.fr
}

\section{RESUME}

La présente étude a pour objectifs de faire des évaluations ethnobotanique et écologique de Jatropha curcas en vue de sa meilleure valorisation au Bénin. Les enquêtes ethnobotaniques ont été réalisées sur 37 groupes ethniques et ont concerné 70\% des Communes du Bénin. La caractérisation écologique a porté sur la description des habitats de l'espèce, sa distribution, ses caractéristiques morphométriques et ses relations avec l'environnement. Les populations utilisent beaucoup plus les feuilles de J. curcas pour traiter les maux de ventre, les maladies du foie, l'ictère, la gonococcie et, le paludisme. La sève est utilisée dans le traitement des morsures de serpents, de la mauvaise haleine et de l'hémorragie. Les graines sont par ailleurs utilisées comme purgatif. Les variables dendrométriques et les niveaux de rendement de l'espèce diffèrent significativement entre individus de différents Départements. L'espèce pousse sur une gamme variée de sol. Les variables abiotiques $\mathrm{pH}_{\text {eau }}, \mathrm{pH}_{\mathrm{kcl}}$, carbone organique ou matière organique n'influencent pas les caractéristiques morphologiques et la productivité de $J$. curcas dans les conditions de production traditionnelle. Toutefois, il a été remarqué que les zones présentant un climat humide et chaud ou celles caractérisées par des sols sableux sont celles dans lesquelles les individus de J. curcas sont peu productifs.

(c) 2009 International Formulae Group. All rights reserved.

Mots clés: Jatropha curcas, connaissances endogènes, diversité écologique, Bénin.

\section{INTRODUCTION}

Malgré la richesse de la biodiversité du continent africain, l'agriculture rencontre de nombreuses difficultés qui entravent son développement économique et le bien-être des populations. La résolution de ces problèmes passe entre autres par la diversification de l'agriculture à travers la valorisation de toutes les ressources et le développement de nouveaux systèmes de production durable. Ceci nécessite une meilleure connaissance du potentiel des ressources naturelles disponibles et déjà intégrées dans les habitudes culturelles des populations rurales. Ainsi, nombreux sont les auteurs qui s'intéressent de plus en plus à l'évaluation et à la valorisation des ressources phytogénétiques. Au nombre de celles-ci, le pourghère ou pignon d'Inde (Jatropha curcas
L.) est une espèce d'Euphorbiaceae à usage multiple utilisée par les populations rurales dans la médecine traditionnelle et comme haie vive dans le bornage des habitations. L'espèce a connu ces dernières années un regain d'intérêt surtout à cause de son utilisation comme biocarburant. En effet, le réchauffement planétaire occasionné par les gaz à effet de serre se manifeste de plus en plus par ses effets néfastes sur le milieu de vie humain ce qui nécessite la recherche de sources d'énergie alternative et renouvelable. L'Europe s'est fixée comme objectif de substituer $5 \%$ de ses utilisations de pétrole par des énergies renouvelables d'ici 2010 (Shanker et al., 2006). En conséquence, nombreuses sont des études qui ont porté ces dernières années sur divers aspects liés à 
l'espèce notamment sur l'impact de sa production sur la dégradation des sols (Podwojewski et al., 2008; Valentin et al., 2008), son écologie (Prasad et al., 2000 ; Qin et al., 2005 ; Gasol et al., 2007 ; Kaushik et al., 2007) et son importance socioéconomique (Gubitz et al., 1999; Zahawi ; 2005 ; Madlener et al., 2006). Il existe très peu d'études qui ont porté sur la caractérisation morpho-génétique de l'espèce (Francis et al., 2005). Les études portant sur la variabilité génétique intra-spécifique ont montré une faible diversité intra population au sein de l'espèce (Basha and Sujatha, 2007; Khaushik et al., 2007; Ranade et al., 2008). Les utilisations de l'espèce comme espèce à multiples usages sont documentées par Heller (1996) et Üllenberg, (2007). Les graines de l'espèce contiennent 27 à 40\% d'huile non comestible, voire toxique, à cause de sa teneur en ester phorbélique (Heller, 1996 ; Üllenberg, 2007). Les graines de J. curcas étaient déjà exportées depuis 1940 du Bénin vers la France où l'huile est extraite et utilisée dans la fabrication du savon de Marseille.

En Inde, l'espèce a une importance capitale et est utilisée dans les plantations dites énergétiques (Shanker et al., 2006). En effet, l'huile issue des graines de $J$. curcas est un excellent carburant, huile lampant et matière première pour la production de savon. Elle est déjà utilisable sans raffinage comme carburant de moteurs diesel à chambre de précombustion, et par transestérification, elle peut être utilisée comme carburant biodiesel par tous les moteurs diesel. J. curcas est une plante sauvage buissonnante de la famille des Euphorbiaceae. Elle est une espèce originaire d'Amérique Centrale introduite sous les tropiques (Arbonnier, 2002). En Afrique, elle est rencontrée dans les terroirs des savanes soudaniennes et des zones guinéennes et s'étend du Sénégal au Cameroun (Arbonnier, 2002).

L'état actuel des connaissances sur Jatropha curcas est encore préliminaire au Bénin. En dehors de sa description dans certaines Flores, très peu, voire pas d'informations sont disponibles sur l'espèce au Bénin. La présente étude vise à combler ce vide et a pour objectifs: (i) d'évaluer les formes de valorisation et les techniques de culture et modes de gestion des plantations de
J. curcas au Bénin, (ii) d'évaluer l'écologie et la variabilité morphologique intraspécifique et, (iii) d'estimer ses rendements en graines.

\section{MATERIEL ET METHODES \\ Etudes ethnobotaniques \\ Choix des localités d'enquête}

L'étude s'est déroulée dans différentes localités situées dans l'ensemble des zones climatiques du Bénin. Il s'agit des localités de la zone guinéo-congolaise $\left(6^{\circ} 25^{\prime} \mathrm{N}-7^{\circ} 30^{\prime} \mathrm{N}\right)$, de la zone soudanoguinéenne $\left(7^{\circ} 30^{\prime} \mathrm{N}-9^{\circ} 45^{\prime} \mathrm{N}\right)$ et de la zone soudanienne $\left(9^{\circ} 45^{\prime} \mathrm{N}-12^{\circ} \mathrm{N}\right) .11$ Départements sur les 12 que compte le Bénin ont été considérés pour la réalisation de l'étude. Le seul Département qui n'a pas été pris en compte est celui du Littoral constitué par la seule ville de Cotonou peu propice à toute activité de plantation à grande échelle. Dans chaque Département échantillonné, 4 ou 5 Communes ont été choisies au hasard pour les investigations. Au total $70 \%$ du total des Communes du Bénin (61 Communes sur 87) ont été échantillonnées pour la conduite de l'étude. Dans chaque Commune, 3 ou 4 villages ont été sélectionnés au hasard.

\section{Enquêtes ethnobotaniques}

Dans chaque village échantillonné, les enquêtes ont été effectuées avec les groupes ethniques (37 au total) des deux sexes et de différentes catégories d'âge. Sous le couvert des chefs de village des différentes localités, les entretiens individuels et/ou de groupe ont été réalisés sur base de questions ouvertes, indirectes et directes et en langue vernaculaire avec ou sans l'aide d'un interprète localement recruté pour la circonstance. Pour plus de précision dans le domaine ethnopharmacologique, les personnes âgées et les guérisseurs ont été individuellement ciblés pour des entretiens. Les informations recueillies étaient relatives (i) à la provenance des plantes, (ii) à l'âge des plantations (iii) aux pratiques culturales (semis, bouturage, gestion des plantations, etc.), (iv) à la valorisation de l'espèce par les populations locales (organes récoltés, transformation des produits, différentes utilisations), et, (v) aux possibilités de mise en place ou non des plantations à une plus grande échelle. 


\section{Analyses des données ethnobotaniques}

Pour le traitement des données, les informations ont été regroupées par Département afin de pouvoir les comparer et proposer à l'échelle nationale des zones d'intérêt pour la valorisation de l'espèce. Après le dépouillement des fiches d'enquête, le taux de réponse par type d'utilisation est exprimé par la formule suivante:

$\mathrm{f}=100 \frac{\mathrm{S}}{\mathrm{N}}$ avec,

$\mathrm{f}$ : taux de réponse; $\mathrm{S}$ : nombre de personnes ayant fourni une réponse positive par rapport à une utilisation donnée; $\mathrm{N}$ : nombre total de personnes interviewées.

Pour tester les relations entre la provenance, la plantation, l'adoption et les Départements, une analyse log-linéaire a été réalisée Caswell (2001). Le modèle saturé dans chaque cas est présenté ci-dessous.

Test des relations entre provenance et Départements:

$\mathrm{LnF}=\mathrm{F}_{\mathrm{a}}+\mathrm{F}_{\mathrm{pr}}+\mathrm{F}_{\mathrm{dp}}+\mathrm{F}_{\mathrm{pr} *} \mathrm{~F}_{\mathrm{dp}}+\varepsilon_{\mathrm{a}}$ ........................(2)

Test des relations entre mode de plantation et Départements:

$\mathrm{LnF}=\mathrm{F}_{\mathrm{b}}+\mathrm{F}_{\mathrm{pl}}+\mathrm{F}_{\mathrm{dp}}+\mathrm{F}_{\mathrm{pl} * \mathrm{~F}_{\mathrm{dp}}}+\varepsilon_{\mathrm{b}}$

Test des relations entre les souhaits d'adoption et Départements:

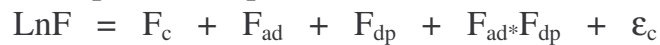

$\mathrm{F}=$ Fréquence globale moyenne ;

$\mathrm{F}_{\mathrm{dp}}=$ Fréquence liée au département ;

$\mathrm{F}_{\mathrm{pr}}=$ Fréquence liée à la provenance ;

$\mathrm{F}_{\mathrm{ad}}=$ Fréquence liée à l'adoption.

Les symboles $\varepsilon_{\mathrm{a}}$, $\varepsilon_{\mathrm{b}}$ et $\varepsilon_{\mathrm{c}}$ représentent les erreurs globales des différents modèles.

Par ailleurs, une Analyse Factorielle des Correspondances a été réalisée pour décrire les relations existantes entre maladies traitées et Départements.

Evaluation écologique de J. curcas au Bénin Distribution de J. curcas et données climatiques des localités d'étude

La distribution de l'espèce a été évaluée à partir des données recueillies dans 232 villages répartis dans 61 Communes et 11 Départements du Bénin. Dans chaque village, il a été évalué la présence/absence de l'espèce. L'espèce est considérée comme présente si elle est retrouvée dans 10 endroits différents $\mathrm{du}$ village au minimum. Les individus retrouvés ont été géoréférencés au Global Positioning System (GPS) permettant ainsi de réaliser, au moyen du logiciel ArcView 3.1, une carte préliminaire de distribution du $J$. curcas au Bénin (Figure 1).

Les données climatiques (pluviométrie moyenne, température normale et l'évapotranspiration potentielle (ETP)) ont été recueillies pour chaque localité d'étude à partir de la station météorologique la plus proche. Ces données ont permis de construire pour chacune d'elle des diagrammes climatiques pour chaque zone climatique (Figure 1).

\section{Évaluations pédologiques des sols sous J. curcas au Bénin}

$\mathrm{Au}$ niveau de chaque station où l'espèce est rencontrée, environ $300 \mathrm{~g}$ d'échantillons par catégorie de sol identifié comme telle, ont été prélevés à une profondeur moyenne de $0-30 \mathrm{~cm}$ au moyen d'une tarière pédologique, à proximité de tous les pieds des individus, pour des analyses au laboratoire de science du sol de la Faculté des Sciences Agronomiques du Bénin. Les caractéristiques analysées ont été la granulométrie (\% argile, \% limon grossier, \% limon fin, $\%$ sable fin, $\%$ sable grossier) par la pipette de Robinson, le $\mathrm{pH}_{\text {eau }}$, le $\mathrm{pH}_{\mathrm{Kcl}}$ dans un rapport sol/solution $=1 / 2.5$, la teneur en matière organique ou en carbone organique par oxydation au $\mathrm{K}_{2} \mathrm{Cr}_{2} \mathrm{O}_{7}$ en milieu acide $\mathrm{H}_{2} \mathrm{SO}_{4}$ (Tableau 1).

\section{Relevés dendrométriques et évaluation des rendements en fruits}

Dans chaque localité d'étude, au moins 10 arbres de $J$. curcas ont été choisis au hasard pour des mesures de diamètre, de hauteur et de rendement en fruits. En outre, pour chaque provenance (Département), des caractéristiques morphométriques (poids moyen, longueur moyenne et diamètre médian moyen), ont été évaluées à partir des mesures prises sur 100 graines choisies au hasard dans un lot issu des Communes du Département considéré. Des analyses de variance et des tests de Newman et Keuls ont permis de comparer au seuil de $5 \%$ les moyennes 


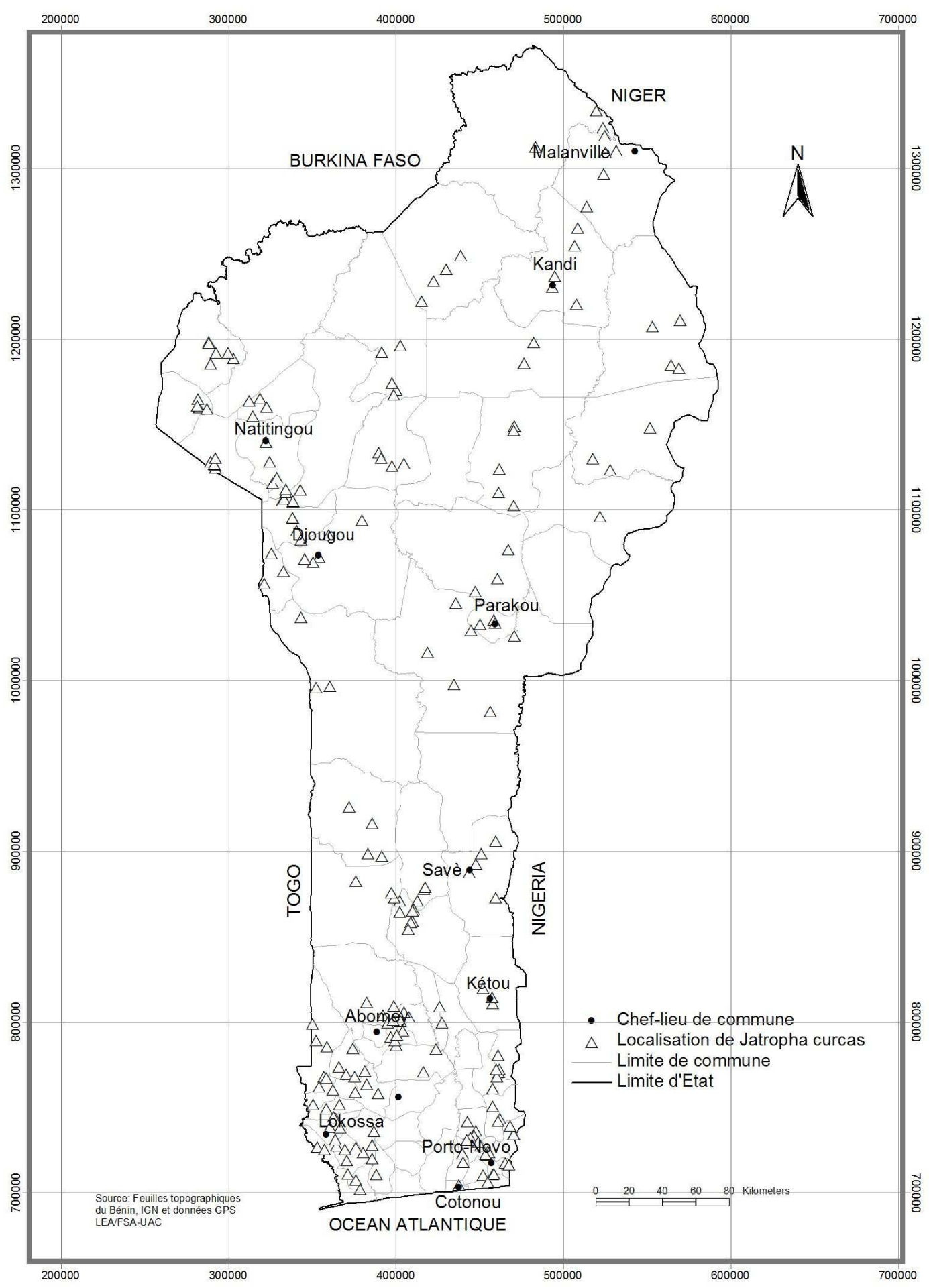

Figure 1: Distribution de Jatropha curcas au Bénin et climatogramme de différentes zones climatiques du Bénin. 
Tableau 1: Caractéristiques moyennes des sols à J. curcas par Département.

\begin{tabular}{lccccccccc}
\hline & \multicolumn{2}{c}{ pH } & \multicolumn{9}{c}{ Granulométrie (\%) } & & \\
\cline { 2 - 8 } Départements & eau & KCl & LG & LF & A & SF & SG & \% Corg & \%MO \\
\hline Collines & 6,88 & 6,61 & 5,5 & 7 & 5,75 & 45,05 & 36,7 & 1,44 & 2,48 \\
Donga & 7 & 6,71 & 2,25 & 3,25 & 12,5 & 40,86 & 41,14 & 1,56 & 2,69 \\
Atacora & 5,49 & 5,2 & 6 & 10,5 & 8 & 39,09 & 36,41 & 1,13 & 1,95 \\
Alibori & 7,1 & 6,76 & 7,5 & 19,5 & 22,25 & 29,94 & 20,81 & 1,8 & 3,1 \\
Atlantique & 5,5 & 5,09 & 0,75 & 7 & 26,5 & 19,35 & 46,41 & 1,02 & 1,76 \\
Ouémé & 7,65 & 7,4 & 8,25 & 20 & 13,25 & 34,68 & 23,82 & 2,53 & 4,36 \\
Plateau & 6,65 & 6,45 & 1,5 & 10 & 11,25 & 11,66 & 65,6 & 1,89 & 3,26 \\
Zou & 6,89 & 6,68 & 0,75 & 2,25 & 11,5 & 21,12 & 64,38 & 1,63 & 2,81 \\
Couffo & 7,18 & 6,95 & 3,75 & 3,5 & 9,5 & 32,94 & 50,31 & 0,83 & 1,43 \\
Mono & 7,42 & 7,08 & 4,25 & 11,75 & 7,75 & 30,39 & 45,87 & 1,43 & 2,47 \\
Borgou & 7,33 & 7,04 & 8,25 & 11,5 & 3,5 & 33,47 & 43,28 & 1,97 & 3,4 \\
\hline
\end{tabular}

Légende : LG Limon Grossier ; LF = Limon fin ; A = argile ; SG = Sable grossier ; SF = Sable fin ; Corg = Carbone organique $; \mathrm{MO}=$ Matière organique.

des différentes variables dendrométriques (hauteur et diamètre) mais aussi les rendements en fruits suivant les différents Départements explorés. Avant de procéder à ces différentes analyses, le test de normalité de Shapiro-Wilk a été effectué pour vérifier la normalité des résidus et le test de BreushPagan (appelé aussi test de Cook-Weisberg) pour vérifier leur homogénéité.

\section{Corrélations entre variables morphologiques et variables environnementales}

En vue de déterminer les corrélations qui existent entre variables morphologiques et environnementales, une Analyse en Composantes Principales (ACP) a été effectuée dans un premier temps sur les variables abiotiques (variables climatiques et édaphiques) avec le logiciel SAS (SAS Inst., 1999). Ensuite, les composantes principales (Zi) obtenues à partir de la combinaison linéaire des variables abiotiques (climatiques et pédologiques) ont été corrélées avec les variables biotiques (dendrométriques et morphométriques) en utilisant le Cœfficient de corrélation de Pearson.

\section{RESULTATS}

Plantation de J. curcas: provenance, gestion culturale et âges

Généralement, les plants de $J$. curcas utilisés proviennent du même village excepté le Département de l'Atacora où ils sont majoritairement $(69,44 \%)$ issus des villages voisins (Tableau 2). En dehors des Départements de l'Atlantique $(15,79 \%)$ et dans une moindre mesure celui du Couffo (5\%), de l'Alibori $(3,45 \%)$ et de l'Atacora $(2,78 \%)$, l'importation des plants et ou des graines des villages plus éloignés est quasi inexistante. L'analyse log-linéaire effectuée indique une différence significative entre les sources d'approvisionnement des plants de $J$. curcas, les populations locales s'approvisionnant plus au niveau local $\left(\chi^{2}=\right.$ $16,47 ; \mathrm{p}=0,003)$. On note par ailleurs une différence significative entre les modes de plantation et les souhaits d'adoption quel que soit le Département $\left(\Delta \mathrm{G}^{2}=8,50 ; \mathrm{p}=0,003\right.$ pour le mode de plantation; $\Delta \mathrm{G}^{2}=5,24 ; \mathrm{p}=$ 0,02 pour l'adoption). Toutefois, il existe une similitude dans les pratiques culturales d'un département à un autre $\left(\Delta \mathrm{G}^{2}=16,61 ; \mathrm{p}=\right.$ 0,06 pour le mode de plantation; $\Delta \mathrm{G}^{2}=7,09$; $\mathrm{p}=0,13$ pour l'adoption). Le Département du Borgou est le seul où la technique de multiplication végétative n'a pas été observée, contrairement aux Départements du Mono $(70 \%)$ et de l'Ouémé $(60 \%)$ où les jeunes branches issues des opérations d'élagage sont directement plantées. Selon les populations, la technique de culture par semis est la meilleure et, très facile pour la reproduction de l'espèce. Toutefois, dans les Départements du Mono et de l'Ouémé où le mode de multiplication végétative est plus adopté, les paysans s'accordent sur le fait que cette technique 
Tableau 2: Gestion des plants de J. curcas par Département.

\begin{tabular}{|c|c|c|c|c|c|c|c|c|}
\hline \multirow[b]{2}{*}{$\begin{array}{l}\text { Département } \\
\text { (n) }\end{array}$} & \multicolumn{3}{|c|}{ Provenance (\%) } & \multicolumn{4}{|c|}{ Mode de plantation (\%) } & \multirow[b]{2}{*}{$\begin{array}{l}\text { \% souhait } \\
\text { d'adoption }\end{array}$} \\
\hline & $\begin{array}{l}\text { Localeme } \\
\text { nt }\end{array}$ & $\begin{array}{l}\text { Autres } \\
\text { Villages }\end{array}$ & $\begin{array}{l}\text { Import } \\
\text { és }\end{array}$ & $\begin{array}{l}\text { Semi } \\
\text { s }\end{array}$ & $\begin{array}{l}\text { Boutura } \\
\text { ge }\end{array}$ & $\begin{array}{l}\text { Semis en } \\
\text { ligne }\end{array}$ & $\begin{array}{l}\text { Plein } \\
\text { Champ }\end{array}$ & \\
\hline Atacora (36) & 27,8 & 69,4 & 2,8 & 72,2 & 50,0 & 52,8 & 50,0 & 97,1 \\
\hline Donga (16) & 75,0 & 25,0 & 0 & 87,5 & 31,3 & 43,8 & 56,3 & 0 \\
\hline Borgou (12) & 100,0 & 0,0 & 0 & 100 & 0 & 100 & 0 & 100 \\
\hline Alibori (29) & 65,5 & 31,0 & 3,5 & 96,6 & 6,9 & 89,7 & 10,3 & 100 \\
\hline Collines (22) & 68,2 & 31,8 & 0 & 90,9 & 36,4 & 63,6 & 36,4 & 0 \\
\hline Zou (22) & 73,7 & 31,6 & 0 & 68,4 & 31,6 & 100 & 0,0 & 94,7 \\
\hline Ouémé (20) & 100 & 0 & 0 & 50 & 60 & 60 & 40,0 & 95 \\
\hline Plateau (16) & 100 & 0 & 0 & 79,0 & 52,6 & 79,0 & 21,1 & 78,9 \\
\hline Couffo (20) & 95,0 & 0 & 5 & 70,0 & 40,0 & 100 & 0 & 100 \\
\hline Mono (20) & 95,0 & 5 & 0 & 50,0 & 70,0 & 100 & 0 & 100 \\
\hline $\begin{array}{l}\text { Atlantique } \\
\text { (19) }\end{array}$ & 94,7 & 0 & 15,8 & 73,7 & 57,9 & 79,0 & 31,6 & 52,6 \\
\hline Global (232) & 76,3 & 22,4 & 2,6 & 75,9 & 40,5 & 77,2 & 24,1 & 79,3 \\
\hline
\end{tabular}

(n) = taille de l'échantillon

favorise une croissance rapide des plantes contrairement aux plantes issues d'un semis direct. Durant les opérations de plantation, le semis en ligne est l'arrangement spatial le plus adopté dans presque tous les Départements, excepté le Département de la Donga où plus de $56 \%$ des paysans pratiquent aléatoirement le mode de semis en plein champ. Le semis en ligne est une technique souvent développée par les paysans pour délimiter leurs espaces (habitations, champs, jardins de case). La plantation se fait sans tenir compte du niveau de fertilité des sols et aucun engrais minéral, ni fumure organique ne sont utilisés. Par ailleurs, 76,29\% des paysans interviewés à travers tout le pays, affirment qu'ils pourront disposer de terres pour la culture de J. curcas si les conditions étaient bien négociées (Tableau 2). Les Départements du Borgou, Alibori, Couffo, Mono, Zou, Atacora, Ouémé, et dans une moindre mesure ceux du Plateau et de l'Atlantique sont les Départements potentiels pour l'adoption de la plantation de $J$. curcas sous forme de système agroforestier. Par contre, dans les Départements des Collines et de la Donga, les paysans interviewés ne semblent pas donner de priorité pour des plantations de l'espèce.
Connaissances ethnobotaniques et utilisations de $J$. curcas au Bénin

$\mathrm{Au}$ Bénin, J. curcas est essentiellement utilisée par les populations locales dans la pharmacopée traditionnelle $(87,50 \%)$ et dans une moindre mesure comme clôture dans la délimitation des habitations $(19,83 \%)$. L'espèce n'est pas utilisée dans l'alimentation et ses organes sont rarement transformés $(2,16 \%)$, commercialisés $(1,29 \%)$ et utilisés comme intrant agricole $(0,43 \%)$. Par ailleurs, les graines sont parfois séchées et utilisées comme source d'énergie à la place du pétrole. Selon certains paysans, l'huile extraite des graines est parfois utilisée pour l'éclairage mais produisant beaucoup plus de fumée.

L'utilisation des différentes parties de la plante dans la pharmacopée traditionnelle constitue un aspect social important dans la vie des communautés rurales (Tableau 3 ).

La majorité des paysans reconnaissent au moins une vertu thérapeutique pour chaque organe ou produit de l'espèce. L'Analyse Factorielle des Correspondances (AFC) effectuée montre qu'avec les deux premiers axes, on explique $62,6 \%$ de l'information sur les maladies traitées par Département.

La figure 2 donne une projection des différentes maladies traitées et Départements sur les deux premiers axes factoriels. L'analyse de cette figure révèle que dans le 
A. E. ASSOGBADJO et al. /Int. J. Biol. Chem. Sci. 3(5): 1065-1077, 2009

Tableau 3 : Importance thérapeutique des organes de J. curcas par Département.

\begin{tabular}{|c|c|c|c|c|c|c|c|c|c|c|c|c|c|}
\hline \multirow{2}{*}{$\begin{array}{l}\text { Produits } \\
\text { utilisés }\end{array}$} & \multirow{2}{*}{\multicolumn{2}{|c|}{$\begin{array}{l}\text { Maladies traitées / } \\
\text { utilisation }\end{array}$}} & \multicolumn{11}{|c|}{ Pourcentage (\%) de répondants par département } \\
\hline & & & $\begin{array}{l}\text { Atlantique } \\
\quad(\mathbf{n}=19)\end{array}$ & $\begin{array}{l}\text { Ouémé } \\
(n=20)\end{array}$ & $\begin{array}{l}\text { Plateau } \\
(n=16)\end{array}$ & $\begin{array}{l}\text { Colline } \\
(n=22)\end{array}$ & $\begin{array}{c}\text { Zou } \\
(\mathbf{n}= \\
22)\end{array}$ & $\begin{array}{c}\text { Couffo } \\
(n=20)\end{array}$ & $\begin{array}{c}\text { Mono } \\
(\mathbf{n}= \\
\mathbf{2 0})\end{array}$ & $\begin{array}{c}\text { Atacora } \\
(n=36)\end{array}$ & $\begin{array}{l}\text { Donga } \\
(\mathrm{n}=16)\end{array}$ & $\begin{array}{l}\text { Borgou } \\
(n=12)\end{array}$ & $\begin{array}{l}\text { Alibori } \\
(n=29)\end{array}$ \\
\hline \multirow[t]{7}{*}{ Feuilles } & $\begin{array}{l}\text { Gonococcie } \\
\text { Syphilis }\end{array}$ & I & 0 & 0 & 0 & 9,09 & 0 & 0 & 0 & 8,33 & 31,25 & 0 & 0 \\
\hline & Rhumatisme & & 0 & 0 & 0 & 4,54 & 4,54 & 0 & 0 & 0 & 0 & 0 & 0 \\
\hline & Mal du foie & & 0 & 0 & 0 & 4,54 & 0 & 0 & 0 & 0 & 0 & 0 & 0 \\
\hline & Ictère & & 5,2 & 10 & 0 & 9,09 & 0 & 0 & 0 & 0 & 0 & 0 & 0 \\
\hline & Maux de ventre & & 21,05 & 0 & 25 & 0 & 0 & 0 & 0 & 11,11 & 0 & 16,66 & 10,34 \\
\hline & Paludisme & & 82,22 & 55 & 100 & 45,45 & 59,09 & 50 & 70 & 27,77 & 100 & 41,66 & 27,58 \\
\hline & Toux & & 0 & 0 & 0 & 13,63 & 0 & 10 & 0 & 5,55 & 0 & 0 & 0 \\
\hline \multirow[t]{4}{*}{ Latex/sève } & Plaie & & 42,10 & 40 & 43,75 & 9,09 & 22,72 & 5 & 0 & 25 & 43,75 & 58,33 & 31,03 \\
\hline & Mauvaise haleine & & 42,10 & 20 & 43,75 & 9,09 & 22,72 & 0 & 15 & 19,44 & 43,75 & 58,33 & 24,13 \\
\hline & Piqûre de serpent & & 0 & 0 & 0 & 0 & 0 & 0 & 0 & 0 & 0 & 16,66 & 0 \\
\hline & Anti-Hémorragie & & 26,31 & 20 & 43,75 & 9,09 & 22,72 & 5 & 0 & 0 & 43,75 & 0 & 31,03 \\
\hline Graines & Purgatif & & 0 & 0 & 0 & 0 & 0 & 5 & 0 & 2,77 & 0 & 0 & 0 \\
\hline Racines / & Ictère & & 15,78 & 5 & 6,25 & 4,54 & 0 & 0 & 0 & 5,55 & 0 & 0 & 0 \\
\hline \multirow[t]{2}{*}{ Branches } & Carie dentaire & & 10,52 & 5 & 18,75 & 13,63 & 9,09 & 10 & 5 & 8,33 & 0 & 0 & 10,34 \\
\hline & $\begin{array}{l}\text { Gonococcie } \\
\text { Syphilis }\end{array}$ & I & 0 & 0 & 0 & 4,54 & 9,09 & 0 & 0 & 0 & 0 & 0 & 0 \\
\hline
\end{tabular}

$\mathrm{n}=$ nombre d'enquêtés. 


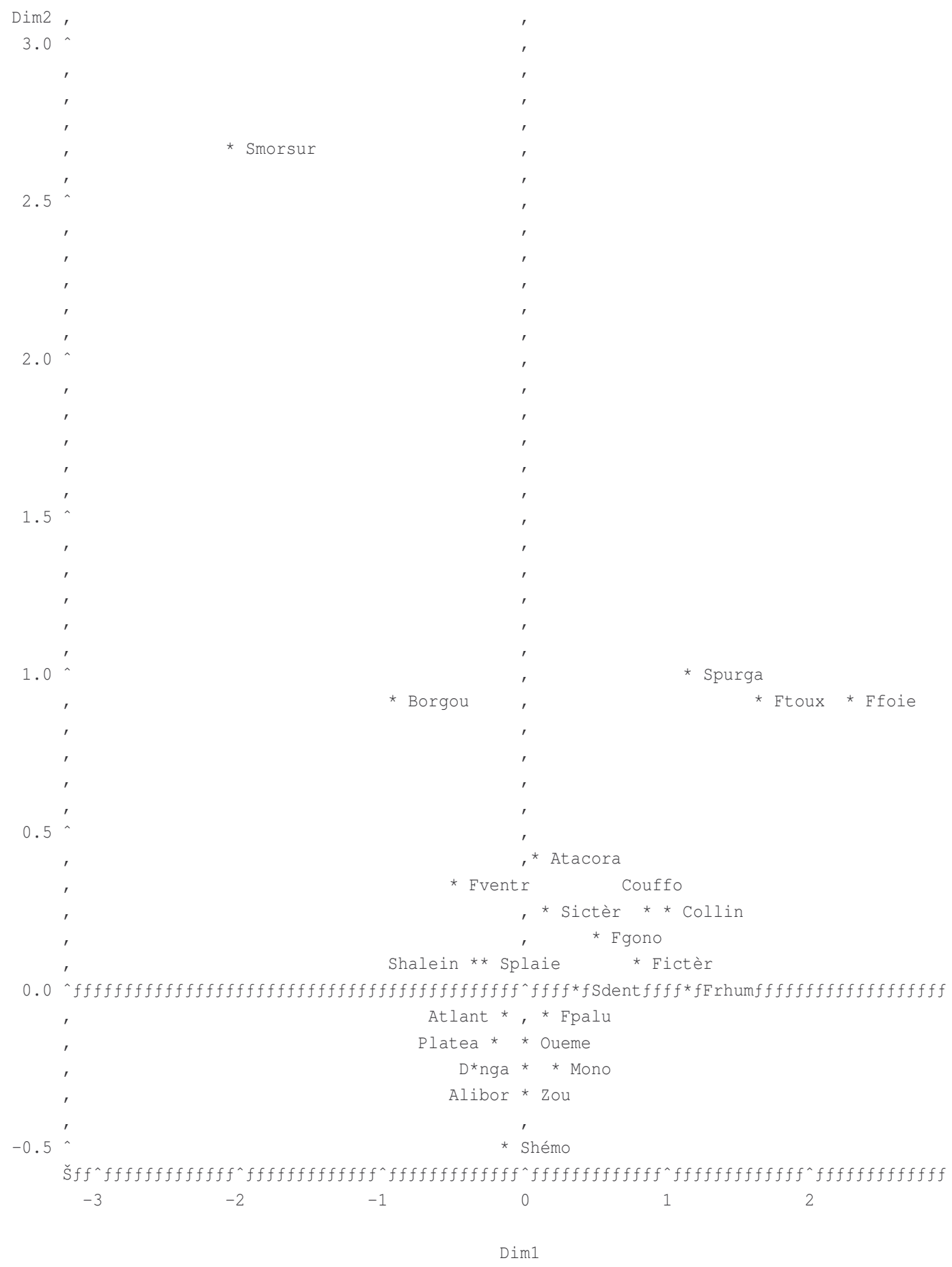

Figure 2: Représentation graphique des différentes maladies traitées et Départements sur les axes factoriels 1 et 2 .

Fgono = gonococie; Frhum = rhumatisme; Ffoie = maladie du foie; Fictèr = ictère traitée avec feuille; Fventr = maux de ventre $;$ Fpalu $=$ paludisme $;$ Ftoux $=$ toux $;$ Shalein $=$ mauvaise haleine $;$ Smorsur $=$ morsure de serpent $;$ Shémo = hémorragie; Spurga $=$ utilisation des graines comme purgatif ; Sdent $=$ carrie dentaire $;$ Ssyphyl $=$ syphylis. 
Département du Borgou, les populations utilisent beaucoup plus la sève de $J$. curcas pour traiter les morsures de serpents. Les autres Départements présentent des tendances similaires quant à l'utilisation des organes de J. Curcas dans le traitement des maladies. On note aussi que les populations utilisent souvent la graine comme purgatif, les feuilles pour la toux et pour guérir les maladies du foie.

Distribution et écologie de $J$. curcas au Bénin

J. curcas est rencontrée dans l'ensemble des Départements et Communes du Bénin. L'espèce est presque toujours plantée et essentiellement pour délimiter des habitations. Généralement, la densité linéaire varie de 3 à 10 plants/m dans presque tous les Départements. En plein champ, la densité moyenne est estimée à environ 2500 pieds/ha. Au Bénin, J. curcas pousse sur une gamme variée de sol (Tableau 1). Elle est rencontrée aussi bien sur des sols acides (Département de l'Atlantique) que neutres (tous les Départements). Les sols sur lesquels évolue l'espèce sont généralement pauvres en matière organique et sont à texture sableuse voire sablo-argileuse ou sablo-limoneuse dans l'ensemble des Départements.

Variabilité morphologique et évaluation des rendements en fruits de $J$. curcas

Quel que soit le paramètre dendrométrique considéré et les niveaux de rendement, il existe une différence significative $(\mathrm{F}=61,34 ; \mathrm{P}<0,001$ pour le diamètre; $\mathrm{F}=9,53 ; \mathrm{P}<0,001$ pour la hauteur $; \mathrm{F}=56,60 ; \mathrm{P}<0,001$ pour le rendement) entre les arbres de différents Départements (Tableau 4). Par ailleurs, les coefficients de variation pour le rendement en fruits des individus de $J$. curcas sont relativement plus élevés, indiquant une variabilité des rendements en fruits d'un arbre à un autre. Le test de Newman et Keuls montre les différents regroupements des Départements selon les grosseurs, les hauteurs et les rendements des arbres (Tableau 4). Les plus gros arbres sont rencontrés dans les Départements du Plateau, de l'Atlantique et $\mathrm{du}$ Zou; les arbres de diamètres intermédiaires sont rencontrés dans les
Départements du Mono, Ouémé, Couffo et Borgou puis enfin les arbres de petits diamètres sont ceux des Départements de la Donga, de l'Alibori, de l'Atacora et des Collines. Pour ce qui est des hauteurs, on note trois groupes constitués respectivement des individus des Départements de l'Ouémé, de l'Atacora, de l'Alibori, du Plateau et du Mono d'une part, des individus des Départements du Zou et de l'Atlantique d'autre part puis enfin des individus des Départements du Borgou, du Couffo, de la Donga et des Collines. Les rendements en fruit mentionnés sont faibles dans les Départements de l'Atlantique, de l'Ouémé, du Plateau, du Zou, du Couffo, du Mono et du Borgou, moyens dans les Départements de la Donga et des Collines puis élevés dans les Départements de l'Atacora et de l'Alibori (Tableau 4).

Relations entre paramètres abiotiques et paramètres morphologiques de $\boldsymbol{J}$. curcas

Les résultats de l'ACP (non présentés dans le document) sur les variables abiotiques montrent qu'avec les 3 premiers axes, on explique $73,7 \%$ des informations sur les paramètres édaphiques et climatiques. Par conséquent, seuls les 3 premiers axes ont été utilisés pour décrire les relations entre les caractéristiques de l'habitat des individus de $J$. curcas. Se basant sur les corrélations entre les différentes variables et les trois axes, on peut conclure que :

- la composante z1 est positivement corrélée avec les variables ETP, pHeau, pHkcl, Carbone organique et Matière organique et négativement avec les variables Pluie et le pourcentage en Sable grossier du sol (SG).

- la composante z2 est positivement corrélée avec les variables humidité relative (HR), Température et le pourcentage en Sable grossier du sol (SG) et négativement corrélée avec le pourcentage en Sable fin du sol (SF).

- la composante z3 est positivement corrélée avec les variables pourcentage en Limon fin (Lf) du sol et en Argile (A) et négativement corrélée avec ETP et le pourcentage en Sable grossier du sol (SG).

Les coefficients de corrélation entre composantes principales et variables biotiques de J. curcas ont révélé que le rendement moyen en fruit des arbres présente une corrélation négative significative avec l'axe 
Tableau 4 : Caractéristiques dendrométriques et rendement en fruits de J. curcas par Département.

\begin{tabular}{|c|c|c|c|c|c|c|}
\hline \multirow[t]{3}{*}{ Départements } & \multicolumn{6}{|c|}{ Caractéristiques dendrométriques } \\
\hline & \multicolumn{2}{|c|}{ Diamètre (cm) } & \multicolumn{2}{|c|}{ Hauteur (m) } & \multicolumn{2}{|c|}{ Nombre de fruits } \\
\hline & Moy & CV (\%) & Moy & CV (\%) & Moy & CV (\%) \\
\hline Atlantique $(\mathrm{n}=34)$ & $30.24^{\mathrm{a}}$ & 71,60 & $2,30^{\mathrm{b}}$ & 56,77 & $13,00^{\mathrm{c}}$ & 248,48 \\
\hline Ouémé $(\mathrm{n}=51)$ & $25.17^{\mathrm{b}}$ & 65,66 & $2,92^{\mathrm{a}}$ & 70,56 & $8,08^{\mathrm{c}}$ & 189,62 \\
\hline Plateau $(n=93)$ & $31.24^{\mathrm{a}}$ & 55,16 & $2,58^{\mathrm{a}}$ & 71,66 & $21,31^{\mathrm{c}}$ & 111,95 \\
\hline Colline $(n=191)$ & $12.72^{\mathrm{c}}$ & 39,49 & $2,01^{\mathrm{c}}$ & 37,90 & $100,52^{\mathrm{b}}$ & 82,44 \\
\hline Zou $(n=146)$ & $29.51^{\mathrm{a}}$ & 43,18 & $2,36^{\mathrm{b}}$ & 33,32 & $33,32^{c}$ & 152,06 \\
\hline Couffo $(n=124)$ & $24.41^{\mathrm{b}}$ & 46,32 & $2,06^{\mathrm{c}}$ & 35,18 & $21,52^{\mathrm{c}}$ & 129,11 \\
\hline Mono $(n=95)$ & $25.77^{\mathrm{b}}$ & 31,13 & $2,52^{\mathrm{a}}$ & 28,58 & $19,86^{\mathrm{c}}$ & 104,93 \\
\hline Atacora $(n=207)$ & $14,00^{\mathrm{c}}$ & 57,38 & $2,77^{\mathrm{a}}$ & 59,01 & $350,49^{\mathrm{a}}$ & 133,51 \\
\hline Donga $(\mathrm{n}=103)$ & $15.26^{\mathrm{c}}$ & 36,97 & $2,05^{\mathrm{c}}$ & 26,46 & $84,08^{\mathrm{b}}$ & 21,81 \\
\hline Borgou $(n=44)$ & $23.80^{\mathrm{b}}$ & 44,71 & $2,20^{c}$ & 29,81 & $23,82^{\mathrm{c}}$ & 134,46 \\
\hline Alibori $(n=67)$ & $14.46^{\mathrm{c}}$ & 60,39 & $2,62^{\mathrm{a}}$ & 57,06 & $377,01^{\mathrm{a}}$ & 284,52 \\
\hline Valeur de F & & 61,34 & & $\mathbf{9 , 5 3}$ & & 56,60 \\
\hline Probabilité & & $<0,001$ & & $<0,001$ & & $<0,001$ \\
\hline
\end{tabular}

$\mathrm{n}=$ nombre d'arbres mesurés ; Moy : moyenne. $\mathrm{CV}=$ Coefficient de Variation ; \% = pourcentage.

Les chiffres présentant en exposant les mêmes lettres dans une même colonne ne sont pas significativement différents $(p>0,001)$ les uns des autres.

z2. De ce fait, le pourcentage en sable fin du sol est un facteur favorable à une meilleure productivité en fruits de l'espèce alors que les valeurs élevées de l'humidité relative, de la température et du pourcentage en sable grossier du sol ont un impact négatif sur le rendement en fruit de J. curcas. Par ailleurs, l'âge est positivement corrélé avec la composante $\mathrm{z} 3$ alors que le diamètre moyen des arbres est négativement corrélé avec la même composante. On en déduit donc que les arbres les plus âgés sont souvent rencontrés sur sols riches en limon fin et peu rencontrés dans les zones à forte évapotranspiration où le pourcentage en sable grossier du sol est élevé. Les sols riches en limon fin et en argile sont défavorables à un développement en diamètre de J. curcas contrairement aux sols riches en sable grossier. Par ailleurs, les variables abiotiques $\mathrm{pH}_{\text {eau }}, \mathrm{pH}_{\mathrm{kcl}}$, Carbone organique ou Matière organique n'influence pas sur les caractéristiques morphologiques ni sur la productivité de J. curcas.

\section{DISCUSSION}

J. curcas est une espèce non alimentaire à usage multiple, plantée par les populations rurales sur toute l'étendue du Bénin. L'utilisation des différentes parties de la plante dans la pharmacopée traditionnelle constitue un aspect social important dans la vie des communautés rurales du Bénin. Les feuilles par exemple sont utilisées dans le traitement du paludisme et les graines comme purgatifs. Les principes actifs contenus dans ces organes de la plante sont donc consommés d'une manière ou d'une autre par les populations. Cependant, certains rapports ont révélé la présence des composées toxiques dans la plante et dans les graines tels que le tannin (10\%), l'acide phytique, l'acide hydrocynamic (HCN), la jatrophine, etc.. (Martinez-Herrera et al., 2006). Il se pose donc des questions de doses admissibles pour préserver la santé des populations. Des études de toxicité et pharmacologiques plus approfondies doivent donc être entreprises pour contribuer à une meilleure valorisation des connaissances ethnobotaniques sur l'espèce.

En outre, J. curcas est une espèce facile à reproduire (par semis et végétativement) et à croissance rapide. Suivant les différentes formes d'utilisation de l'espèce et le niveau actuel de production, nous pouvons déduire que très peu de marchés potentiels s'offrent à l'état actuel à une valorisation économique de l'espèce au Bénin. Cependant, au niveau international, il se développe de plus en plus de nouveaux marchés rémunérateurs pour la vente des différents organes de l'espèce notamment les 
graines qui sont utilisées dans la production de biocarburant comme alternative aux sources d'énergie non renouvelables. D'ici l'an 2020, l'Inde va employer environ 5 millions de personnes pour travailler sur plus de 10 millions d'ha de plantation de J. curcas. Ainsi, elle sera en mesure de fournir annuellement sur le marché mondial de biocarburant 7,5 millions de tonnes de biodiesel (Shanker et al., 2006). En outre, il est observé au Sénégal et au Mali, des efforts soutenus pour une production à grande échelle de l'espèce. On déduit de tout ce qui précède l'existence de marchés potentiels régionaux et internationaux qui devraient inciter à une plus grande valorisation de l'espèce.

Au Bénin, 76,29\% des paysans interviewés affirment qu'ils pourront disposer de terres pour la culture de J. curcas si les conditions étaient bien négociées. Si les biocarburants peuvent être profitables pour nos agriculteurs, ils représentent également une formidable opportunité de créer une véritable agro-industrie génératrice d'activités dans nos provinces. Pour presser l'huile de Jatropha, la transformer en biodiesel il faudra mettre en place ces petites industries qui aujourd'hui manquent cruellement aux régions reculées du pays. Ceci permettra de créer non seulement des emplois agricoles, mais également les emplois nécessaires à la transformation de ces produits.

Par ailleurs, il est démontré que $3 \mathrm{~kg}$ de graines de $J$. curcas sont susceptibles de fournir $1 \mathrm{~L}$ d'huile (Business \& Financial Times, 2007). En outre, en fonction du type de sol, du climat et des modes de gestion culturale, 1 ha de plantation de $J$. curcas peut générer jusqu'à 4 tonnes de graines (Business $\&$ Financial Times, 2007). On en déduit que 1 ha de plantation de $J$. curcas peut potentiellement fournir $1333 \mathrm{~L}$ d'huile brute. Il est donc indispensable de développer une agriculture qui produira à la fois de l'énergie et des aliments puisque l'espace à utiliser pour les cultures alimentaires traditionnelles et le Jatropha n'est pas le même. Le Jatropha nous permettra de détourner les fonds utilisés à l'achat de carburant vers nos agriculteurs et grâce aux variétés non toxiques, son tourteau riche en protéines permettra de réaliser des aliments équilibrés pour nos élevages. Pour un meilleur développement de la filière Jatropha au Bénin, il faudra aussi assurer aux paysans une formation adéquate sur les différents modes de gestion optimale des plantations, disposer d'une main d'oeuvre conséquente et de grands espaces de préférence dans des zones dégradées peu propices à l'agriculture. Cependant, il faudra garder à l'esprit qu'une telle activité si très lucrative pourrait à long terme engendrer des concurrences avec des espaces réservés aux cultures vivrières. Ceci pourrait affecter la sécurité alimentaire des ménages qui ne disposeraient plus d'assez d'espaces pour l'agriculture. Un autre risque potentiel d'inciter les paysans à se rallier à une telle initiative serait que ces derniers ne soient pas impliqués dans la fixation des prix de cession qui peuvent évoluer à la baisse dans le temps. Les conditions du marché et les situations macroéconomiques mondiales peuvent amener à moyen ou à long terme les raffineries à ne plus s'intéresser à l'achat des graines chez ces paysans. L'espèce n'étant pas alimentaire, il leur sera alors difficile de développer des stratégies pour écouler les produits. A moins que des stratégies de valorisation au niveau local soient développées simultanément pour produire l'huile à partir des graines en vue de complémenter les sources d'énergie non renouvelable qui seraient localement utilisées.

Il a été observé que $J$. curcas pousse sur des sols aussi bien acides que neutres pauvres en carbone organique. Les variables abiotiques $\mathrm{pH}_{\text {eau }}, \mathrm{pH}_{\mathrm{kcl}}$, Carbone organique et Matière organique n'influencent pas les caractéristiques morphologiques et la productivité de J. curcas. L'espèce présenterait alors une certaine plasticité telle que observée également par Prasad et al. (2000) et Gasol et al. (2007); elle offre par conséquent la possibilité de son introduction dans l'ensemble des zones climatiques et agroécologiques du Bénin. Le Jatropha peut donc pousser sur des terres marginales impropres à l'agriculture. Le Jatropha permettrait donc de valoriser des terres aujourd'hui mises à nu par la déforestation et qui ne sont pas utilisées pour l'agriculture vivrière. Il a été remarqué que les zones présentant un climat plus humide et chaud ou celles caractérisées par des sols pauvres en sable fin sont celles dans lesquelles les individus de J. curcas sont peu productifs. Par 
ailleurs, Prasad et al. (2000) ont montré que le développement racinaire de l'espèce est entravée sur des sols lourds. Aussi, Kaushik et al. (2007), Qin et al. (2005) ont signalé que $J$. curcas ne devrait jamais être plantée à forte capacité de rétention en eau comme les vertisols et autres sols argileux. Ainsi, le développement de stratégies de propagation de l'espèce avec comme objectif la production de grandes quantités de fruits, devrait viser les localités où les températures sont relativement basses et celles moins humides avec des sols riches en sable fin. Les localités du Nord du Bénin et dans une moindre mesure celles du Centre-Bénin étant respectivement localisées dans le Soudanien et le Soudano-Guinéen seraient donc plus propices à un meilleur rendement de l'espèce. Cependant, J. curcas peut provoquer l'érosion des sols et la dégradation des éléments nutritifs (Podwojewski et al., 2008; Valentin et al., 2008). En conséquence, il n'est pas conseillé de proposer la plantation de l'espèce sur des sols riches propices à l'agriculture.

Des difficultés probables qui peuvent aussi limiter le développement de la filière au Bénin seraient les problèmes de subvention des petits entrepreneurs et de renforcement des capacités des paysans à une meilleure gestion des plantations, les problèmes liés au foncier et aux feux de végétation (surtout dans le domaine soudanien) et le manque d'un cadre légal sur les biocarburants comme ce fut le cas à Madagascar (Üllenberg, 2007).

Les propriétés médicinales reconnues aux différents organes de l'espèce font d'eux des sources potentielles pour la valorisation en pharmacopée traditionnelle. Ces mêmes propriétés sont reconnues à l'espèce dans d'autres pays tels que le Ghana, Madagascar et en Inde (Üllenberg, 2007 ; Jongschaap et al., 2007). Cependant, le marché des produits médicinaux est très compétitif et exige la mise en conformité à des normes internationales. Il se pose alors un problème de définition des normes (harmonisation à des normes exigées pour les produits médicinaux, gestion de la qualité) pour chacun des produits afin de favoriser leur exportation vers les marchés plus rémunérateurs notamment ceux de l'Union Européenne. En attendant, les traitements pharmacologiques à base de ces produits sont localement utilisés et contribuent à la sécurité sanitaire des populations rurales.

\section{Conclusion}

En somme, le développement des activités de production de J. curcas dépend essentiellement des conditions locales, des potentialités et des marchés disponibles. Une subvention de la part de la communauté internationale et des gouvernements est nécessaire pour accompagner l'activité. Enfin, des études d'impacts intégrant les dimensions humaines, environnementales, économiques, sociales et physiques sont indispensables avant tout développement d'une telle activité au Bénin.

\section{REFERENCES}

Arbonnier M. 2002. Arbres, Arbustes et Lianes des Zones Sèches d'Afrique de l'Ouest ( $2^{\text {ème }}$ édn). CIRAD: Paris, France.

Basha SD, Sujatha M. 2007. Inter and intrapopulation variability of Jatropha curcas (L.) characterized by RAPD and ISSR markers and development of populationspecific SCAR markers. Euphytica, 156: 375-386.

Business \& Financial Times. 2007. Farmers reject Jatropha Posted on: 25-Apr-2007, Ghana

Caswell H. 2001. Matrix Population Models: Construction Analysis and Interpretation (2nd edn). Sinauer Associates.

Francis G, Edinger R, Becker K. 2005. A concept for simultaneous wasteland reclamation, fuel production, and socioeconomic development in degraded areas in India: need, potential and perspectives of Jatropha plantations. Natural Resources Forum, 29(1): 12-24.

Gasol CM, Gabarrell X, Anton A, Rigola M, Carrasco J, Ciria P. 2007. Life cycle assessment of a Brassica carinata bioenergy cropping system in southern Europe. Biomass Bioenergy, 31(8): 543555.

Gubitz GM, Mittelbach M, Trabi M. 1999. Exploitation of the tropical oil seed plant Jatropha curcas L. Bioresource Technol., 67(1): 73-82.

Heller J. 1996. Physic nut. Jatropha curcas L. Promoting the conservation and use of 
underutilized and neglected crops. 1. Institute of Plant Genetics and Crop Plant Research, Gatersleben/ International Plant Genetic Resources Institute, Rome.

Jongschaap REE, Corré WJ, Bindraban RS, Brandenburg WH. 2007. Claims and Facts on Jatropha curcas L. Global Jatropha curcas evaluation, breeding and propagation. Report 158, PRI, WUR, The Netherland.

Kaushik N, Kumar K, Kumar S, Kaushik N, Roy S. 2007. Genetic variability and divergence studies in seed traits and oil content of jatropha (Jatropha curcas L.) accessions. Biomass \& Bioenergy, 31: 497-502.

Madlener R, Robledo C, Muys B, Blanco Freja JT. 2006. A sustainability framework for enhancing the long-term success of LULUCF Projects. Climatic Change, 75(1-2): 241-271.

Martinez-Herrera J, Siddhuraju P, Francis G, Da vila-Ortı G, Becker K. 2006 Chemical composition, toxic/ antimetabolic constituents, and effects of different treatments on their levels, in four provenances of Jatropha curcas L. from Mexico. Food Chemistry, 96: 8089.

Podwojewski P, Orange D, Jouquet P, Valentin C, Thiet NV, Janeau, JL, Toan TD. 2008. Influence of land use changes on runoff and erosion from micro-plots and a catchment in northern Vietnam. Catena, 24: 109-118.

Prasad CMV, Krishna MVSM, Reddy CP, Mohan KR. 2000. Performance evaluation of non-edible vegetable oils as substitute fuels in low heat rejection diesel engines. Proceedings of the Institution of Mechanical Engineers Part D - J Auto Eng 214(Part D):181-187.
Qin W, Ming-Xing H, Ying X, Xin-Shen Z, Fang C. 2005. Expression of a ribosome inactivating protein (curcin 2) in Jatropha curcas is induced by stress. Journal of Biosciences, 30: 351-357.

Ranade SA, Srivastavaa AP, Ranab TS, Srivastavaa J, Tulia R. 2008. Easy assessment of diversity in Jatropha curcas L. plants using two single-primer amplification reaction (SPAR) methods. Biomass \&Bioenergy, 32: 533 - 540 .

SAS Inst. 1999. SAS/STAT User's Guide. SAS Institute, Cary, NC. Business \& Financial Times 2007. Farmers reject Jatropha. Posted on: 25-Apr-2007, Ghana.

Shanker C, Dhyani SK. 2006. Insect pests of Jatropha curcas L. and the potential for their management. Current Science, 91: 2-25.

Üllenberg A. 2007. Jatropha à Madagascar Rapport sur l'état actuel du secteur GTZ Agresti A., Analysis of categorical data. New York, USA 1990.

Valentin C, Agus F, Alamban R, Boosaner A, Bricquet JP, Chaplot V, de Guzman T, de Rouw A, Janeau JL, Orange D, Phachomphonh K, Phai Do, Podwojewski P, Ribolzi O, Silvera N, Subagyono K, Thiébaux J, Toan T, Vadari T. 2008. Runoff and sediment losses from 27 upland catchments in Southeast Asia: Impact of rapid land use changes and conservation practices. Agriculture, Ecosystems and Environment, 128: 225-238.

Zahawi RA. 2005. Establishment and growth of living fence species: an overlooked tool for the restoration of degraded areas in the tropics. Restoration Ecololy, 13(1): 92-102. 